\title{
ABORDAGEM DO PACIENTE COM HEMORRAGIA DIGESTIVA ALTA NÃO VARICOSA
}

\author{
MANAGEMENT OF PATIENTS WITH NONVARICEAL UPPER GASTROINTESTINAL HEMORRHAGE
}

Karla Glaysia A. Lourenço ${ }^{1}$ \& Ricardo B. de Oliveira ${ }^{2}$

${ }^{1}$ Pós-graduanda. ${ }^{2}$ Docente. Divisão de Gastroenterologia. Departamento de Clínica Médica. Faculdade de Medicina de Ribeirão Preto-USP. CorResPondêncIA: Dra. Karla Glaysia A. Lourenço. Departamento de Clínica Médica. Hospital das Clínicas da FMRP/USP. Cep: 14048800 .Ribeirão Preto - SP. Tel: (16) 6022457 . karlaglaysia@hotmail.com

LOURENÇO KG \& OLIVEIRA RB. Abordagem do paciente com hemorragia digestiva alta não varicosa. Medicina, Ribeirão Preto, 36: 261-265, abr./dez. 2003.

RESUMO - A hemorragia digestiva alta não varicosa é uma emergência comum, e continua sendo uma causa significativa de morbimortalidade. Esta revisão objetiva oferecer aos estudantes de Medicina e médicos recomendações para o atendimento do paciente com esse tipo hemorragia, de forma a mostrar as abordagens terapêuticas, reunindo opiniões de diferentes especialistas.

UNITERMOS - Hemorragia Gastrointestinal.

\section{1- INTRODUÇÃO}

A hemorragia digestiva é uma emergência comum, com expressivas taxas de morbidade e mortalidade. Em que pesem os progressos da prática da Medicina, nas últimas décadas, o declínio dessas taxas foi desprezível ${ }^{(1)}$. A hemorragia digestiva alta (HDA) representa um ônus elevado para o Sistema de Saúde. Nos Estados Unidos, a prevalência da HDA é de, aproximadamente, 170 casos por 100000 de adultos, por ano, com um custo total estimado em $750 \mathrm{mi}$ lhões de dólares ${ }^{(2)}$.

Em nosso meio, a HDA não varicosa, isto é, aquela que não se deve a sangramento de varizes esofágicas, tem, como causa mais comum, a doença ulcerosa péptica, responsável por $50 \%$ a $70 \%$ dos casos. A despeito dos recentes avanços na terapia, principalmente dos relacionados às novas técnicas de hemostasia endoscópica, a taxa de mortalidade tem se mantido, essencialmente, inalterada, entre $6 \%$ e $8 \%$, o que pode ser explicado pelo fato de os pacientes estarem mais velhos e apresentarem co-morbidades numerosas ${ }^{(2)}$.

Diretrizes para a abordagem da HDA têm sido desenvolvidas por grupos de estudiosos do problema. Esta revisão, dirigida a estudantes de Medicina e médicos, tem o objetivo de apresentar as diretrizes que visam otimizar o atendimento do paciente com hemorragia digestiva alta.

\section{2- DIRETRIZES PARA O MANEJO DE PACI- ENTES COM HDA NÃO VARICOSA}

\section{1- Normatização do atendimento}

Os hospitais devem elaborar protocolos específicos para o atendimento de HDA. Esses protocolos devem definir, precisamente, papéis para os diferentes profissionais envolvidos, além de prever acesso à endoscopia de urgência e com profissionais treinados em hemostasia endoscópica ${ }^{(3)}$. 


\section{2- Avaliação e conduta iniciais}

A avaliação imediata e a recomposição da volemia apropriada são críticas para o manejo adequado dos pacientes com hemorragia digestiva alta. A magnitude da redução da volemia pode ser avaliada a partir da pressão arterial e da freqüencia de pulso.(Tabela I) Trabalhos recentes mostraram que medidas intensivas, precoces, de ressuscitação resultam na diminuição no lapso entre a admissão e a estabilização hemodinâmica, que se associa à redução da mortalidade $^{(4)}$. O conjunto de medidas que deve constituir $\mathrm{o}$ atendimento inicial de pacientes com HDA encontra-se na Tabela II.

\section{3- Uso de sonda nasogástrica}

Em pacientes selecionados (por ex., na impossibilidade de endoscopia digestiva alta), o uso de sonda nasogástrica pode ser considerado, com o intuito de confirmar hemorragia do trato digestivo superior, pela caracterização do conteúdo aspirado. Além dis- so, os achados podem ter valor prognóstico: a presença de sangue vivo, no aspirado, revela sangramento ativo, que se associa a maiores riscos ${ }^{(3)}$.

\section{4- Estratificação de risco}

O sangramento cessa sem recorrência, após admissão, em $80 \%$ dos pacientes com HDA. A maioria da morbidade e a mortalidade concentram-se entre os $20 \%$ restantes, cujo sangramento não cessa ou recorre. Diante disso, a estratificação clínica dos pacientes em alto e baixo risco para ressangramento é importante na definição da conduta médica ${ }^{(3)}$. Além disso, outros fatores de risco existem, como idade avançada e comorbidades.

Por isso, foram elaboradas normas de quantificação de risco (escores), associando-se achados clínicos e endoscópicos, que permitem a separação dos pacientes com HDA em alto e baixo risco ${ }^{(3)}$. O escore mais usado, atualmente, é o de Rockall (Tabelas III e IIIA $)^{(4,5)}$.

Tabela I - Mensuração da perda sanguínea

Magnitude da perda

Pequena (20\% volemia)

Moderada (20-40\% volemia)

Maciça (> 40\% volemia)
P. A.

Decúbito: normal Em pé: queda $<14 \mathrm{mmHg}$

$90-100 \mathrm{mmHg}$ ou queda $>14 \mathrm{mmHg}$
Pulso

Decúbito: normal Em pé: $\uparrow<20$ bpm

$100-120$ bpm

$>120 \mathrm{bpm}$
Perda

$<1000 \mathrm{~mL}$

$1500 \mathrm{~mL}$

$>2000 \mathrm{~mL}$

\section{Tabela II - Atendimento inicial do paciente com HDA}

Avaliação clínica

Acesso venoso

Recomposição da volemia

Exames de urgência
Determinar o estado hemodinâmico e a urgência, verificar palidez, temperatura de extremidades, enchimento do leito ungueal, estigmas de hepatopatia, pulso e pressão arterial (Ver Tabela I)

Veia periférica, se os parâmetros forem normais; duas veias periféricas, se parâmetros forem anormais, mas, pressão arterial for normal em decúbito; a veia central, sempre que possível, se perda maciça.

Reposição imediata de volume com soro fisiológico ou Ringer-lactato. Soluções de reposição, compostas não oferecem qualquer vantagem, encarecem o tratamento, sobrecarregam a equipe de enfermagem e sua manufatura embute risco de erro. Concentrado de hemácias, se anemia ou perda maciça.

Hematócrito e/ou hemoglobina, tipo sanguíneo e Rh; plaquetas e coagulograma, se evidência de hepatopatia ou doença hematológica; uréia e creatinina, se choque circulatório (em idosos sempre). 
Tabela III - Escore de Rockall, para ressangramento na HDA

\begin{tabular}{|c|c|c|c|c|}
\hline \multirow{2}{*}{ Variáveis } & \multicolumn{4}{|c|}{ Pontuação } \\
\hline & 0 & 1 & 2 & 3 \\
\hline Idade & $<60$ & $60-79$ & 80 & - \\
\hline Estado da volemia & não & Pulso $>100 \mathrm{bpm}$ & $\mathrm{PA}<100 \mathrm{mmHg}$ & - \\
\hline Doenças associadas & - & - & $\begin{array}{l}\text { ICC, insuficiência coronariana. } \\
\text { Outras doenças graves }\end{array}$ & $\begin{array}{l}\text { Insuficiência renal } \\
\text { ou hepática, } \\
\text { carcinomatose }\end{array}$ \\
\hline Diagnóstico & $\begin{array}{l}\text { Mallory-Weiss, sem } \\
\text { estigma, sem lesões }\end{array}$ & $\begin{array}{l}\text { Todos os outros } \\
\text { diagnósticos }\end{array}$ & Câncer gastrintestinal & - \\
\hline Estigma endoscópico & $\begin{array}{l}\text { Ausente ou coágulo } \\
\text { plano }\end{array}$ & - & $\begin{array}{l}\text { Sangue no trato gastrintestinal } \\
\text { alto; coágulo aderido, vaso } \\
\text { visível ou sangramento ativo }\end{array}$ & - \\
\hline
\end{tabular}

Tabela IIIA - Escore de Rockall, para ressangramento na HDA

\begin{tabular}{|c|c|c|c|c|}
\hline Pontuação & $\begin{array}{c}\text { Número de } \\
\text { pacientes }\end{array}$ & Ressangramento & $\begin{array}{c}\text { Mortalidade em pacientes } \\
\text { com ressangramento }\end{array}$ & $\begin{array}{c}\text { Mortalidade } \\
\text { em geral }\end{array}$ \\
\hline$<2$ & $30 \%$ & $4,3 \%$ & $0 \%$ & $0,1 \%$ \\
\hline $3-5$ & $48 \%$ & $14 \%$ & $2,5 \%$ & $4,6 \%$ \\
\hline $6-8$ & $22 \%$ & $37 \%$ & $14 \%$ & $22 \%$ \\
\hline
\end{tabular}

Características clínicas, preditoras de elevado risco de ressangramento, demandando a observação rigorosa e/ou prolongada ${ }^{(3)}$.

- Idade > 65 anos

- Choque (perda maciça)

- Co-morbidades (cardiorrespiratória, renal, hepática, coagulopatia)

- Baixos níveis de $\mathrm{Hb}$

- Recorrência de melena

- Necessidade de hemotransfusões

- Sangue vivo no toque retal, no vômito ou na sonda nasogástrica

- Tempo de protrombina alongado

- Pacientes hospitalizados por outras razões, como sepse, na presença de aumento de uréia e creatinina ou transaminases

Estigmas endoscópicos, preditores de ressangramento são ${ }^{(6)}$.

- Hemorragia ativa, vaso visível (Forrest I e II)
- Tamanho da úlcera ( $>2 \mathrm{~cm})$

- Sítio de sangramento (parede posterior da primeira porção duodenal, curvatura gástrica menor).

Os dois primeiros achados endoscópicos, referidos podem ser compilados na classificação de Forrest, que considera os itens relacionados a seguir.

\section{I- Hemorragia ativa}

Ia. Hemorragia ativa, em jato

Ib. Hemorragia ativa, lenta, em porejamento

\section{II- Hemorragia recente}

IIa. Presença de vaso visível, não sangrante

IIb. Presença de coágulo na base da lesão

IIc. Presença de hematina na base da lesão

\section{III- Sem hemorragia}

Presença de lesão com base limpa, sem evidências de sangramento. 


\section{5- Exame endoscópico}

A endoscopia digestiva alta (EDA), precoce (nas primeiras $24 \mathrm{~h}$ ) é valiosa por permitir maior segurança para a classificação do risco. Além disso, alguns estudos randomizados revelaram que a EDA precoce e a terapêutica endoscópica reduzem a necessidade de hemotransfusões, o tempo de permanência hospitalar e os custos com os pacientes ${ }^{(3)}$.

\section{6- Terapêutica endoscópica}

Existem vários métodos de terapêutica endoscópica, tais como: injeção de substâncias, método térmico, mecânico (hemoclipes) e outros (como o Laser) ${ }^{(7)}$.

\subsection{1- Soluções usadas na terapêutica por inje- ção}

- Álcool absoluto

- Etanolamina(1 a 5\%)

- Polidocanol a $1 \%$

- Adrenalina (pura-1:10000; com NaCl normal ou hipertônico; com esclerosantes; prévia aos térmi$\cos )$

- Trombina com $\mathrm{NaCl}$

- Água destilada

- Solução de glicose hipertônica.

\subsection{2- Terapêutica térmica}

- Heater probe

- Eletrocoagulação mono/multipolar

- Eletrocoagulação com gás de argônio

Não há demonstração clara de superioridade de uma das soluções de injeção sobre as demais, o mesmo ocorrendo em relação às outras técnicas. $\mathrm{O}$ método recomendável é aquele com o qual o endoscopista esteja familiarizado e tenha bons resultados ${ }^{(8)}$. No entanto, a terapia combinada (por ex: injeção de uma substância associada a um método térmico) mostrou-se superior à monoterapia, na hemostasia, com reduções estatisticamente significativas na taxa de ressangramento ${ }^{(3)}$.

\section{7- Vigilância endoscópica}

A repetição de EDA de rotina não é recomendada. Pode ser benéfica em pacientes selecionados e de alto risco, ou quando o exame endoscópico foi in- completo ou inconclusivo por razões técnicas (por exemplo, presença de coágulos, impedindo a visualização), ou nos casos de ressangramento após tratamento endoscópico, prévio $^{(3)}$.

\section{8- Indicações de cirurgia}

A indicação cirúrgica precoce pode ser particularmente necessária em cada caso relacionado a seguir $^{(3,9)}$.

- Persistência da hemorragia com dificuldade de manutenção da estabilidade hemodinâmica

- Falha de retratamento endoscópico

- Necessidade de hemotransfusão de metade ou mais da volemia estimada nas primeiras $24 \mathrm{~h}$

- Em pacientes com mais de 60 anos e ou co-morbidades graves, com instabilidade hemodinâmica

O tratamento endoscópico possui algumas limitações em relação ao sucesso terapêutico, indicandose a intervenção cirúrgica, precocemente. É recomendado não se insistir no retratamento endoscópico, nas seguintes situações ${ }^{(8)}$

- Úlceras de difícil acesso ao endoscópio

- Úlceras com hemorragia maciça de vaso calibroso, localizado na parede posterior da primeira porção duodenal ou na curvatura menor do corpo gástrico proximal

- Úlceras profundas e calosas.

\section{9- Farmacoterapia}

Antagonistas do receptor $\mathrm{H}_{2}$ da histamina: não são recomendados no manejo de pacientes com HDA. Vários estudiosos concluíram que não há melhora estatisticamente significante, quando comparados a outros fármacos ou à terapia endoscópica ${ }^{(3)}$.

Somatostatina e octreotide: não são recomendados na conduta de rotina dos pacientes com HDA não varicosa. Entretanto, é terapia que pode ser usada em pacientes com sangramento incontrolável, enquanto aguardam EDA, nos que esperam por cirurgia ou naqueles que a cirurgia é contra-indicada ${ }^{(3)}$.

Inibidor de bomba de prótons (IBP): o uso endovenoso em bolus, seguido de infusão contínua mostrou ser eficaz em diminuir o ressangramento dos pacientes com sucesso da terapêutica endoscópica. No entanto, os trabalhos demonstram a superioridade da combinação de fármacos e hemostasia endoscópica em relação apenas ao tratamento medicamentoso ${ }^{(3)}$. A sugerida dose eficaz seria de $80 \mathrm{mg}$, por via endo- 
venosa, em bolus de IBP, seguida de $8 \mathrm{mg} / \mathrm{h}$, por $72 \mathrm{~h}$ após terapia endoscópica. Outros estudos ressaltam que a terapia com altas doses de IBP deve ser considerada nos pacientes que aguardam EDA, podendo as doses serem administradas, por via oral, nos pacientes de baixo risco, e, por via endovenosa, nos pacientes de alto risco ${ }^{(3)}$.

Recomenda-se que seja pesquisada a presença de Helicobacter pylori no momento da endoscopia pelo teste da urease ou pela histologia, pois a sua erradicação reduz o índice de ressangramento a longo $\operatorname{prazo}^{(8)}$.

Pacientes, considerados de baixo risco para morbimortalidade e para ressangramento, podem receber alta, após EDA, em 24 h ${ }^{(3)}$; ou seja, esses pacientes podem deixar o hospital imediatamente após a estabilização clínica. Além disso, pacientes com Forrest III podem iniciar a alimentação oral logo após a endoscopia.

LOURENÇO KG \& OLIVEIRA RB. Management of patients with nonvariceal upper gastrointestinal hemorrhage. Medicina, Ribeirão Preto, 36: 261-265, apr./dec.2003.

ABSTRACT - Nonvariceal upper gastrointestinal hemorrhage is a common emergency, and continues to be a significant cause of morbidity and mortality. This review aimed to medical student and doctors offers guidelines for the approach of patients with this type of hemorrhage, by showing therapeutic options involving different specialists' reports.

UNITERMS - Gastrointestinal Hemorrhage.

\section{REFERÊNCIAS BIBLIOGRÁFICAS}

1 - HUANG CS \& LICHTENSTEIN DR. Nonvariceal upper gastrointestinal bleeding. Gastroenterol Clin North Am 32: 1053-78, 2003.

2 - LEUNG JW \& CHUNG SSC. Tratamento prático do sangramento gastrintestinal alto não-varicoso. In: TYTGAT GNJ \& CLASSEN M. Prática de endoscopia terapêutica. Revinter, Rio de Janeiro, p.1-16, 2000.

3 - BARKUN A; BARDOU M \& MARSHALL K. Consensus Recommendations for Managing Patients with Nonvariceal Upper Gastrointestinal Bleeding. Ann Intern Med 139: 843-857, 2003.

4 - BARADARIAN R; RAMDHANEY S; CHAPALAMADUGU R; SKOCZYLAS L; WANG K;RIVILIS S; REMUS K; MAYER I \& ISWARA KT. Early intensive resuscitation of patients with upper gastrointestinal bleeding decreases mortality. Am J Gastroenterol 99: 619-623, 2004.
5 - ROCKALL TA; LOGAN RFA \& DEVLIN HB. Risk assessment after acute upper gastrointestinal hemorrhage. Gut 38: 316$321,1996$.

6 - LUNA LL; VARGAS C; LUNA RA \& JUNQUEIRA DPR. Endoscopia digestiva na hemorragia alta não varicosa. In: SOCIEDADE BRASILEIRA DE ENDOSCOPIA DIGESTIVA. Endoscopia digestiva. Medsi, Rio de Janeiro, p.228-58, 2000.

7 - MOURA EG; SAKAI P \& MALUF FILHO F. Hemorragia digestiva alta não varicosa. In: SAKAI P; ISHIOKA S \& FAUZE FILHO M. Tratado de endoscopia digestiva diagnóstica e terapêutica. Atheneu, São Paulo, vol. II, p.195-208, 2001.

8 - SAKAI P; VARGAS C; MAGUILNIK I; SILVA MB; MASCARENHAS R; RITTER R \& KUGA R. Consenso Brasileiro em Endoscopia Digestiva da Sociedade Brasileira de Endoscopia Digestiva (SOBED). GED 21: 33-37, 2002.

9 - LÁZARO SA. Hemorragia digestiva. In: Cirurgia de urgência. Editora Medsi, Rio de Janeiro, p.310-326, 1994. 